\title{
Prevalência de alterações posturais em escolares do ensino médio em uma cidade no Sul do Brasil ${ }^{1}$
}

\author{
Cíntia Detsch, ${ }^{2}$ Anna Maria Hecker Luz, ${ }^{2}$ Cláudia Tarragô \\ Candotti, ${ }^{3}$ Daniela Scotto de Oliveira, ${ }^{3}$ Franciane Lazaron, ${ }^{3}$ \\ Lisiane Kiefer Guimarães ${ }^{4}$ e Patrícia Schimanoski ${ }^{5}$
}

Como citar Detsch C, Luz AMH, Candotti CT, Scotto de Oliveira D, Lazaron F, Guimarães LK, et al. Prevalência de alterações posturais em escolares do ensino médio em uma cidade no Sul do Brasil. Rev Panam Salud Publica. 2007;21(4):231-8.

RESUMO Objetivos. Estimar a prevalência de alterações posturais laterais e ântero-posteriores em adolescentes do sexo feminino e verificar se determinados fatores socioeconômicos, demográficos, antropométricos e comportamentais estão associados a essas alterações posturais.

Métodos. Inquérito epidemiológico com amostra representativa de 495 estudantes do ensino médio regular diurno, com idade de 14 a 18 anos na Cidade de São Leopoldo, Brasil. As estudantes foram avaliadas nos meses de outubro e novembro de 2004. As alterações posturais foram definidas como alterações nas curvas fisiológicas da coluna vertebral, identificadas através de avaliação postural não-invasiva.

Resultados. Observou-se uma prevalência de 66\% (IC95\%: 61,5 a 70,0) para as alterações laterais e de 70\% (IC95\%: 65,2 a 73,5) para as alterações ântero-posteriores. As alterações laterais foram mais prevalentes nas alunas com índice de massa corporal normal (razão de prevalência, ou RP = 1,32; IC95\%: 1,09 a 1,59) e nas que assistiam à televisão por mais de 10 horas semanais $(R P=1,16$; IC95\%: 1,02 a 1,32). A prevalência de alterações ântero-posteriores foi maior nas alunas cujos responsáveis estudaram até o nível fundamental (responsáveis femininos, $R P=1,30$; IC95\%: 1,09 a 1,55; e responsáveis masculinos, $R P=1,20 ;$ IC95\%: 1,02 a 1,40) e nas alunas com sobrepeso ou obesidade ( $R P=1,33 ;$ IC95\%: 1,19 a 1,48).

Conclusões. Preocupa a alta prevalência de alterações na postura das adolescentes, uma vez que essas alterações podem gerar problemas na coluna vertebral a médio e longo prazo. É importante que profissionais da área da saúde, inclusive professores de educação física, estejam aptos a realizar avaliações posturais, e que esse procedimento seja realizado rotineiramente nas escolas.

Palavras-chave Coluna vertebral, postura/epidemiologia, saúde do adolescente, saúde escolar, Brasil.

1 Parte da dissertação de mestrado de Cíntia Detsch, apresentada em 2005 ao Programa de PósGraduação em Saúde Coletiva da Universidade do Vale do Rio dos Sinos (UNISINOS), São Leopoldo (RS) Brasil.

2 Universidade do Vale do Rio dos Sinos (UNISINOS), Programa de Pós-Graduação em Saúde Coletiva. Enviar correspondência para Cíntia Detsch no seguinte endereço: Rua Felipe dos Santos 77/ apto 1004 C, Padre Réus, CEP 93020-180, São Leopoldo, RS, Brasil. Fone: +55-51-3591-4028; e-mail: cintiadetsch@gmail.com

3 Universidade do Vale do Rio dos Sinos (UNISINOS), Curso de Educação Física.

4 Universidade do Vale do Rio dos Sinos (UNISINOS), Curso de Nutrição.

5 Universidade do Vale do Rio dos Sinos (UNISINOS), Curso de Fisioterapia.
As doenças nas costas, mais especificadamente as dores na região lombar (lombalgia), são muito prevalentes na população adulta (1) e também na população adolescente (2). Muitas vezes, a ocorrência da lombalgia precede ou é 
concomitante às alterações da postura corporal. Essa associação pode ser explicada pelo fato de que muitas posturas corporais adotadas no dia-a-dia são inadequadas para as estruturas anatômicas, pois aumentam o estresse total sobre os elementos do corpo, especialmente sobre a coluna vertebral, podendo gerar desconfortos, dores ou incapacidades funcionais (3).

Muitos problemas posturais, em especial aqueles relacionados com a coluna vertebral, têm sua origem no período de crescimento e desenvolvimento corporais, ou seja, na infância e na adolescência $(4,5)$. Além disso, durante essas fases, os indivíduos estão sujeitos a comportamentos de risco para a coluna, principalmente aqueles relacionados à utilização de mochilas e à postura sentada (para assistir à televisão e utilizar o computador, por exemplo) (6). Tais comportamentos podem acarretar alterações posturais tanto laterais como ântero-posteriores (7).

Assim, a identificação dos padrões posturais de crianças e adolescentes passa a ser preponderante para a prevenção das alterações na postura corporal, sejam elas funcionais ou estruturais. Alguns países desenvolvidos já adotam a realização sistemática de avaliações posturais durante a fase escolar para identificar e acompanhar a progressão das alterações da postura em geral e, principalmente, da postura da coluna vertebral (4).

Entretanto, existe controvérsia sobre as avaliações posturais, uma vez que seus resultados geram apenas indícios da existência da alteração. Tradicionalmente, o diagnóstico clínico tem sido realizado através de exames radiológicos, que permitem conhecer a severidade da alteração na coluna. Contudo, o uso de radiografias em estudos de prevalência e incidência expõe a população aos efeitos da radiação, sendo considerado inapropriado por ser antiético e envolver um custo muito alto (8). Desse modo, as avaliações posturais nas quais os indivíduos são submetidos a testes nãoinvasivos tornam-se uma opção viável para estudos das alterações da postura corporal em populações.

Sob o ponto de vista ortopédico, as avaliações posturais em escolares são benéficas, pois proporcionam uma oportunidade individual de diagnóstico precoce. Muitas vezes, essa é a única oportunidade que a criança ou o adolescente tem de obter um diagnóstico da sua postura e informações sobre a saúde de sua coluna vertebral (4).

O objetivo deste estudo foi estimar a prevalência de alterações posturais laterais e ântero-posteriores em adolescentes do sexo feminino e verificar se tais alterações estão associadas a determinados fatores socioeconômicos, demográficos, antropométricos e comportamentais.

\section{MATERIAIS E MÉTODOS}

Trata-se de um inquérito epidemiológico de base escolar, realizado nos meses de outubro e novembro de 2004 com alunas de 14 a 18 anos de idade da Cidade de São Leopoldo, Estado do Rio Grande do Sul, Brasil. A amostra foi calculada utilizando-se o programa Epi Info 6.0 e tendo por base uma população de 9721 escolares do sexo feminino nessa faixa etária (9). A escolha do sexo feminino foi baseada no princípio de que, na adolescência, a incidência de alterações posturais é maior nas meninas em relação aos meninos (10).

Foram calculados vários tamanhos de amostra, pois este estudo fez parte de uma pesquisa mais ampla, com diferentes desfechos em saúde. Para a presente análise, a amostra escolhida foi aquela selecionada para o estudo sobre prevalência de dores nas costas, que foi a maior das amostras calculadas (11). O estudo sobre dores nas costas foi analisado em outro relatório. Os dados utilizados para o cálculo foram: prevalência de $30 \%$, nível de confiança de $95 \%$, erro amostral de $5 \%$, efeito de delineamento de 1,5 e perdas e recusas de $10 \%$. Quinhentas e quinze adolescentes de todas as escolas de ensino médio regular diurno de São Leopoldo (10 privadas e 10 públicas) foram convidadas a participar do estudo, sendo que 495 delas aceitaram participar. A recusa de 20 adolescentes equivale a $4 \%$, o que não gerou viés de seleção, uma vez que foi inferior aos $10 \%$ rela- tivos a perdas e recusas acrescentados no cálculo para tamanho da amostra.

A seleção da amostra foi proporcional ao número de alunas em cada escola, garantindo a cada unidade amostral a mesma probabilidade de pertencer à amostra. Em cada escola realizou-se um sorteio aleatório simples do total das alunas de 14 a 18 anos regularmente matriculadas no terceiro trimestre letivo diurno.

O estudo foi aprovado pelo Comitê de Ética em Pesquisa da Universidade do Vale do Rio dos Sinos (UNISINOS). As alunas sorteadas receberam um termo de consentimento livre e esclarecido para leitura, decisão de participação e assinatura dos responsáveis.

Para a coleta dos dados, foram realizados os seguintes procedimentos: avaliação da postura corporal, medição da estatura e da massa corporal e aplicação de um questionário sobre variáveis que podem estar associadas à postura. A avaliação postural foi realizada num posturógrafo, com as alunas na posição ortostática, com os pés descalços, cabelos presos e trajando roupas apropriadas para a avaliação (calça corsário ou short colado ao corpo e top ou biquíni). Cada aluna foi avaliada individualmente, uma única vez, pela mesma avaliadora, em sua escola de origem. Nessa avaliação, os passos realizados foram os seguintes: a) localização (por palpação) e marcação dos acrômios direito e esquerdo, das espinhas ilíacas póstero-superiores (EIPS) direita e esquerda, dos ângulos inferiores das escápulas direita e esquerda, do processo espinhoso da sétima vértebra torácica e da fossa anterior do maléolo lateral direito; b) medição bilateral das alturas dos acrômios e das EIPS em relação ao solo, utilizando-se um antropômetro; c) medição da distância total entre os ângulos inferiores das escápulas e das distâncias de cada ângulo inferior em relação ao processo espinhoso correspondente, utilizando-se uma régua com nível acoplado para assegurar a horizontalidade da medida; d) aplicação do teste de Adams, para verificar a existência de gibosidade; e) registro fotográfico de costas e de perfil da postura das alunas no posturógrafo, 
utilizando-se máquina fotográfica digital (Olympus D-390, 2 megapixels) posicionada sobre um tripé a $2 \mathrm{~m}$ da parede onde o posturógrafo foi fixado e a uma altura de 1,48 m do solo.

Para o registro fotográfico, as alunas foram orientadas a permanecer na postura em pé usual do dia-a-dia, com os braços ao longo do corpo e o peso do corpo distribuído igualmente sobre os dois pés paralelos. Para avaliação das alterações laterais, a aluna foi posicionada de costas em relação à avaliadora, com olhar fixo para o posturógrafo e com os dois pés a uma mesma distância da linha central do posturógrafo. Para avaliação das alterações ântero-posteriores, a aluna foi posicionada com o lado direito voltado para a avaliadora e a posição dos pés ajustada de modo que a fossa anterior do maléolo lateral direito ficasse alinhada com a linha central da base do posturógrafo. As avaliações foram conduzidas em locais que não apresentavam desnível de solo.

Para verificar a consistência da avaliação da pesquisadora e a confiabilidade dos instrumentos de coleta de medidas, foi realizado um estudo piloto em duplicata, com intervalo de 2 semanas entre as coletas de dados, com 20 alunas (4\% da amostra). Os resultados do piloto em duplicata foram submetidos ao teste kappa. Para as alterações laterais, o kappa foi de 0,87 ( $P=$ $0,01)$. Para as alterações ântero-posteriores, o kappa foi de 0,70 ( $P=0,007)$. Esses resultados mostram a consistência e a confiabilidade do protocolo de avaliação postural.

As variáveis socioeconômicas, demográficas e comportamentais foram coletadas por questionário auto-aplicável padronizado, codificado e testado, contendo questões fechadas. Nas questões referentes às posturas ao assistir à televisão e trabalhar no computador, foram apresentadas figuras indicativas de posturas corretas e incorretas e as alunas marcavam a postura que melhor correspondesse ao seu dia-a-dia.

A mensuração das variáveis antropométricas (estatura e massa corporal) foi realizada para possibilitar o cálculo do índice de massa corporal (IMC). A medição da estatura foi realizada com um antropômetro fixado na parede e ajustado com um nível, para evitar erro de posicionamento do mesmo, e os valores aferidos em centímetros. A medição da massa corporal foi realizada em balança digital (Rib Plenna), calibrada com um peso padronizado de $5 \mathrm{~kg}$ a cada cinco alunas pesadas. Para análise, o IMC foi agrupado segundo a classificação de Cole et al. (12). As características socioeconômicas, demográficas, antropométricas e comportamentais foram agrupadas para análise das variáveis de associação.

Para a avaliação postural, foram utilizados os dados coletados a partir das medidas das alturas e distâncias dos segmentos corporais, teste de gibosidade, observação e análise das fotos no computador. Em cada fotografia foi desenhado um fio de prumo, a partir do referencial de Kendall et al. (13). As alunas foram divididas em dois grupos: sem alteração e com alteração na postura.

Em relação às alterações laterais, as alunas sem alteração de postura foram aquelas que passaram pelo teste do fio de prumo (pontos anatômicos marcados coincidentes com o prumo) ou que apresentaram apenas um dos seguintes itens: a) cabeça inclinada à direita ou esquerda; b) assimetria na altura dos acrômios; ou c) assimetria na altura das EIPS. As alunas com alteração de postura foram aquelas que apresentaram dois ou mais dos seguintes itens: a) não passar no teste do fio de prumo; b) assimetria na altura dos acrômios; c) assimetria na altura das EIPS; d) assimetria do triângulo de Talles; ou e) presença de gibosidade.

Em relação às alterações ânteroposteriores, as alunas sem alteração de postura foram aquelas que passaram no teste do fio de prumo ou que apresentaram apenas um entre dois itens - cabeça anteriorizada ou ombros e escápulas protraídos - acompanhado ou não de a) pelve antevertida ou retrovertida; b) abdômen proeminente; ou c) corpo deslocado para frente ou para trás.

As alunas com alteração de postura foram aquelas que apresentaram dois ou mais entre quatro itens - a) não passar no teste do fio de prumo; b) ca- beça anteriorizada; c) ombros e escápulas protraídos; e d) curvatura dorsal aumentada - acompanhados ou não de dois ou mais dos seguintes itens: a) pelve antevertida ou retrovertida; b) abdômen proeminente; ou c) curvatura lombar aumentada ou diminuída. Essa classificação foi baseada em parâmetros propostos na literatura $(13,14)$.

A entrada dos dados foi realizada por duas digitadoras independentes no programa Epi Info 6.0 para identificação de erros de digitação e sua correção. Para a análise bivariada, foi utilizado o Statistical Package for the Social Sciences (SPSS) 11.0. Para análise multivariada, utilizou-se o programa Stata 7.0.

A análise bivariada incluiu o teste do qui-quadrado $\left(\chi^{2}\right)$ e o cálculo das razões de prevalência com seus intervalos de confiança de 95\% (IC95\%) separadamente para os desfechos alterações posturais laterais e ânteroposteriores, para verificar a associação entre as variáveis do estudo. As variáveis dependentes foram as alterações posturais laterais e ântero-posteriores. As variáveis independentes foram o tipo de escola e a escolaridade dos responsáveis (variáveis socioeconômicas); a idade (variável demográfica); o IMC (variável antropométrica); e a postura com bolsa ou mochila, a postura em sala de aula, a postura ao assistir à televisão, o número de horas semanais assistindo à televisão, a postura ao usar computador e o número de horas semanais de uso de computador (variáveis comportamentais).

Foram incluídas no modelo multivariado as variáveis que apresentaram significância de $P<0,20$, consideradas como diferenciais de risco. A análise multivariada, visando a confirmar as associações entre as variáveis do estudo, seguiu o modelo da figura 1 . Nesse modelo, a decisão acerca das variáveis a serem incluídas na análise seguiu a hierarquia na relação entre elas. As variáveis pertencentes ao bloco I foram as primeiras a serem incluídas no modelo, seguidas das variáveis do bloco II, todas determinando alterações posturais.

A permanência das variáveis no modelo final exigiu significância estatística de $P<0,05$ no teste de Wald em 
FIGURA 1. Modelo hierárquico de análise para alterações posturais

\begin{tabular}{ll} 
Bloco I & \\
$\begin{array}{l}\text { Variáveis socioeconômicas } \\
\text { - Tipo de escola }\end{array}$ & $\begin{array}{l}\text { Variável demográfica } \\
\text { - Escolaridade da responsável feminina }\end{array}$ \\
- Escolaridade do responsável masculino & \\
\hline Bloco II & \\
$\begin{array}{l}\text { Variável antropométrica } \\
\text { - Índice de massa corporal }\end{array}$ & Variável comportamental \\
\hline Desfecho & Atividades da vida diária \\
\hline
\end{tabular}

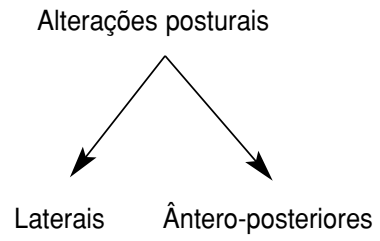

pelo menos uma categoria. Os modelos multivariados foram construídos separadamente para cada um dos dois desfechos (alterações posturais laterais e ântero-posteriores) e o modelo de regressão utilizado foi o de Poisson.

\section{RESULTADOS}

Os resultados das características socioeconômicas, demográficas, antropométricas e comportamentais são apresentados nas tabelas 1 e 2 . A maioria das adolescentes estudava em escolas públicas. Observou-se uma prevalência de $66 \%$ para as alterações posturais laterais e de $70 \%$ para as alterações ântero-posteriores.

Segundo a análise bivariada, as alterações laterais estiveram associadas ao IMC e ao número de horas semanais assistindo à televisão. As alunas com IMC normal apresentaram maior prevalência de alterações se comparadas com aquelas com sobrepeso ou obesidade. As alunas que assistiam à televisão por mais de 10 horas semanais apresentaram maior prevalência de alterações em relação às que não assistiam à televisão ou que a assistiam por menos de 10 horas semanais. As alu- nas que assistiam à televisão por mais tempo apresentaram maior prevalência de postura incorreta ao assistir à televisão se comparadas com as que assistiam à televisão por menos de 10 horas por semana (tabela 1).

As alterações posturais ântero-posteriores estiveram associadas com $\mathrm{O}$ tipo de escola, com a escolaridade dos responsáveis e com o IMC. As estudantes de escolas públicas apresentaram maior prevalência de alterações quando comparadas com as estudantes de escolas privadas; as alunas cujos responsáveis (tanto femininos quanto masculinos) estudaram até o nível fundamental apresentaram maior prevalência de alterações se comparadas com as alunas cujos responsáveis estudaram até o nível superior ou pósgraduação. As alunas com sobrepeso ou obesidade tiveram maior prevalência de alterações do que as alunas com IMC normal (tabela 2).

$\mathrm{Na}$ análise multivariada, as alterações posturais laterais continuaram associadas com o IMC $(P=0,006)$ e com o número de horas semanais assistindo à televisão $(P=0,028)$. As alterações posturais ântero-posteriores continuaram associadas com as mesmas variáveis da análise bivariada, principalmente com a escolaridade da responsável feminina $(P=0,005)$ e com o IMC $(P<0,001)$.

\section{DISCUSSÃO}

A prevalência de alterações posturais, tanto laterais quanto ânteroposteriores, foi alta em nosso estudo. O mesmo foi observado em um estudo realizado no Estado da Bahia com estudantes de educação física de ambos os sexos, com média de idade de 22 anos, avaliados com o posturógrafo. Naquele estudo, a prevalência de alterações laterais foi de $69,6 \%$, e de alterações ântero-posteriores, de 41,9\%. Separando-se os resultados por sexo, o grupo feminino apresentou uma prevalência de $72,7 \%$ de alterações laterais (15), um percentual próximo ao encontrado no presente estudo.

Utilizando o posturógrafo, o fio de prumo e o teste de Adams, pesquisadores do Estado da Paraíba encontraram uma prevalência ainda mais alta de alterações posturais entre homens e mulheres com média de idade de 29 anos. Os resultados indicaram que, em relação às mulheres, 96\% delas apresentaram algum tipo de alteração postural lateral, e que $74 \%$ apresentaram algum tipo de alteração postural ântero-posterior (16).

Prevalências mais baixas foram apontadas por outros estudos. No Estado de Santa Catarina, escolares de ambos os sexos, com idade entre 10 e 16 anos, foram avaliados com auxílio do posturógrafo e do fio de prumo. Esse estudo observou uma prevalência de 28,2\% de alterações ântero-posteriores entre os avaliados (17). Em outra pesquisa, com avaliações utilizando o teste de Adams, foi apontada uma prevalência de $48,4 \%$ de alterações posturais laterais nas alunas de 10 a 12 anos de idade, e de 49,5\% de alterações laterais nas alunas de 13 a 15 anos (18). Outro estudo, também utilizando o fio de prumo, porém com escolares mais novos (6 a 15 anos de idade), de ambos os sexos, encontrou uma prevalência de $38,88 \%$ de alterações laterais e uma prevalência de 33,27\% de alterações ântero-posteriores (19). 
TABELA 1. Prevalência de alterações posturais laterais em estudantes do sexo feminino com idade de 14 a 18 anos, São Leopoldo, Estado do Rio Grande do Sul, Brasil, 2004

\begin{tabular}{|c|c|c|c|c|}
\hline Variáveis & No. (\%) & $\begin{array}{c}\text { Alterações } \\
\text { laterais } \\
\text { No. }(\%)\end{array}$ & $P^{a}$ & $\begin{array}{l}\text { Razão de } \\
\text { prevalência }\end{array}$ \\
\hline \multicolumn{5}{|l|}{ Socioeconômicas } \\
\hline \multicolumn{5}{|l|}{ Tipo de escola } \\
\hline Privada & $134(27,1)$ & $87(64,9)$ & & 1,00 \\
\hline Pública & $361(72,9)$ & $239(66,2)$ & 0,790 & $1,02(0,88$ a 1,17$)$ \\
\hline \multicolumn{5}{|l|}{ Escolaridade da responsável femininac ${ }^{c}$} \\
\hline Até nível superior ou pós-graduação & $102(21,4)$ & $72(70,6)$ & & 1,00 \\
\hline Até nível médio & $145(30,5)$ & $95(65,5)$ & & $0,93(0,78$ a 1,10$)$ \\
\hline Até nível fundamental & $229(48,1)$ & $149(65,1)$ & 0,370 & $0,92(0,79$ a 1,08$)$ \\
\hline \multicolumn{5}{|l|}{ Escolaridade do responsável masculinoc ${ }^{c}$} \\
\hline Até nível superior ou pós-graduação & $111(23,8)$ & $73(65,8)$ & & 1,00 \\
\hline Até nível médio & $146(31,3)$ & $97(66,4)$ & & $1,01(0,85$ a 1,21$)$ \\
\hline Até nível fundamental & $209(44,9)$ & $136(65,1)$ & 0,869 & $0,99(0,84$ a 1,17$)$ \\
\hline \multicolumn{5}{|l|}{ Demográfica } \\
\hline \multicolumn{5}{|l|}{ Faixa etária (anos) } \\
\hline 17 a 18 & $138(27,9)$ & $86(62,3)$ & & 1,00 \\
\hline 16 & $176(35,5)$ & $116(65,9)$ & & $1,06(0,89$ a 1,25$)$ \\
\hline 14 a 15 & $181(36,6)$ & $124(68,5)$ & 0,251 & $1,10(0,93$ a 1,29$)$ \\
\hline \multicolumn{5}{|l|}{ Antropométrica } \\
\hline \multicolumn{5}{|l|}{ Índice de massa corporal } \\
\hline Normal & $387(78,2)$ & $269(69,5)$ & & 1,00 \\
\hline Sobrepeso/obesidade & $108(21,8)$ & $57(52,8)$ & $0,001^{b}$ & $0,76(0,63$ a 0,92$)$ \\
\hline \multicolumn{5}{|l|}{ Comportamentais } \\
\hline \multicolumn{5}{|l|}{ Postura com bolsa ou mochilac ${ }^{c}$} \\
\hline Correta (vestindo duas alças ou transpassada) & $273(55,7)$ & $172(63,0)$ & & 1,00 \\
\hline Incorreta (mochila pendendo em um só ombro) & $217(44,3)$ & $151(69,6)$ & 0,127 & $1,10(0,97$ a 1,25$)$ \\
\hline \multicolumn{5}{|l|}{ Postura em sala de aula } \\
\hline Correta (pés e costas apoiados) & $50(10,1)$ & $36(72,0)$ & & 1,00 \\
\hline Incorreta (outras) & $445(89,9)$ & $290(65,2)$ & 0,334 & $0,91(0,75$ a 1,09$)$ \\
\hline \multicolumn{5}{|l|}{ Postura ao assistir à televisão ${ }^{c}$} \\
\hline Correta (pés e costas apoiados) & $36(7,4)$ & $21(58,3)$ & & 1,00 \\
\hline Incorreta (outras) & $453(92,6)$ & $302(66,7)$ & 0,309 & $1,14(0,86$ a 1,52$)$ \\
\hline \multicolumn{5}{|l|}{ Horas assistindo à televisão por semana } \\
\hline 0 a 10 & $334(67,5)$ & $209(62,6)$ & & 1,00 \\
\hline mais de 10 & $161(32,5)$ & $117(72,7)$ & $0,026^{b}$ & $1,16(1,02$ a 1,32$)$ \\
\hline \multicolumn{5}{|l|}{ Postura ao usar computador ${ }^{c}$} \\
\hline Correta (pés e costas apoiados) & $111(27,5)$ & $75(67,6)$ & & 1,00 \\
\hline Incorreta (outras) & $292(72,5)$ & $192(65,8)$ & 0,731 & $0,97(0,83$ a 1,13$)$ \\
\hline \multicolumn{5}{|l|}{ Horas de uso do computador por semana } \\
\hline 0 a 6 & $410(82,8)$ & $266(64,9)$ & & 1,00 \\
\hline Mais de 6 & $85(17,2)$ & $60(70,6)$ & 0,312 & $1,09(0,93$ a 1,27$)$ \\
\hline
\end{tabular}

${ }^{a}$ Análise bivariada. Teste do qui-quadrado.

${ }^{\mathrm{b}}$ Associação estatística significativa $(P<0,05)$.

${ }^{c}$ Somente para as alunas às quais a variável se aplica.

A variação nos resultados desses estudos possivelmente se deve às diferenças nas faixas etárias das amostras $\mathrm{e}$ às diferentes metodologias utilizadas, isoladas ou combinadas entre si, para avaliação postural. Apesar das diferenças entre as prevalências apontadas, todos os estudos mostram uma realidade preocupante em relação à saúde de crianças, adolescentes e adultos, pois as alterações posturais modi- ficam os pontos de pressão sobre os segmentos corporais e podem causar problemas como dores nas costas, se não no momento atual, provavelmente em uma idade mais avançada (20).

Em relação aos fatores associados às alterações posturais, o presente estudo apresenta a limitação de não possibilitar o estabelecimento de uma relação temporal de causa e efeito, devido ao tipo de delineamento utili- zado, no qual as informações sobre exposição (variáveis socioeconômicas, demográficas, antropométricas e comportamentais) e desfecho (alterações posturais laterais e ântero-posteriores) foram obtidas ao mesmo tempo. Apesar disso, o estudo demonstrou algumas associações entre as variáveis coletadas.

Dentre as variáveis estudadas, independentemente de ser a análise biva- 
TABELA 2. Prevalência de alterações posturais ântero-posteriores em estudantes do sexo feminino com idade de 14 a 18 anos, São Leopoldo, Estado do Rio Grande do Sul, Brasil, 2004

\begin{tabular}{|c|c|c|c|c|}
\hline Variáveis & No. (\%) & $\begin{array}{c}\text { Alterações } \\
\text { ântero-posteriores } \\
\text { No. (\%) }\end{array}$ & $P^{a}$ & $\begin{array}{l}\text { Razão de } \\
\text { prevalência }\end{array}$ \\
\hline \multicolumn{5}{|l|}{ Socioeconômicas } \\
\hline \multicolumn{5}{|l|}{ Tipo de escola } \\
\hline Privada & $134(27,1)$ & $78(58,2)$ & & 1,00 \\
\hline Pública & $361(72,9)$ & $266(73,7)$ & $0,001^{b}$ & $1,27(1,08$ a 1,48$)$ \\
\hline \multicolumn{5}{|l|}{ Escolaridade da responsável feminina ${ }^{c}$} \\
\hline Até nível superior ou pós-graduação & $102(21,4)$ & $60(58,8)$ & & 1,00 \\
\hline Até nível médio & $145(30,5)$ & $91(62,8)$ & & $1,07(0,87$ a 1,31$)$ \\
\hline Até nível fundamental & $229(48,1)$ & $175(76,4)$ & $0,000^{b}$ & $1,30(1,09$ a 1,55$)$ \\
\hline \multicolumn{5}{|l|}{ Escolaridade do responsável masculino ${ }^{C}$} \\
\hline Até nível superior ou pós-graduação & $111(23,8)$ & $71(64,0)$ & & 1,00 \\
\hline Até nível médio & $146(31,3)$ & $88(60,3)$ & & $0,94(0,87$ a 1,14$)$ \\
\hline Até nível fundamental & $209(44,9)$ & $160(76,6)$ & $0,006^{b}$ & $1,20(1,02$ a 1,40$)$ \\
\hline \multicolumn{5}{|l|}{ Demográfica } \\
\hline \multicolumn{5}{|l|}{ Faixa etária (anos) } \\
\hline 17 a 18 & $138(27,9)$ & $98(71,0)$ & & 1,00 \\
\hline 16 & $176(35,5)$ & $121(68,8)$ & & $0,97(0,84$ a 1,12$)$ \\
\hline 14 a 15 & $181(36,6)$ & $125(69,1)$ & 0,725 & $0,97(0,84$ a 1,12$)$ \\
\hline \multicolumn{5}{|l|}{ Antropométrica } \\
\hline \multicolumn{5}{|l|}{ Índice de massa corporal } \\
\hline Normal & $387(78,2)$ & $251(64,9)$ & & 1,00 \\
\hline Sobrepeso/obesidade & $108(21,8)$ & $93(86,1)$ & $0,000^{b}$ & $1,33(1,19$ a 1,48$)$ \\
\hline \multicolumn{5}{|l|}{ Comportamentais } \\
\hline \multicolumn{5}{|l|}{ Postura com bolsa ou mochilac } \\
\hline Correta (vestindo duas alças ou transpassada) & $273(55,7)$ & $192(70,3)$ & & 1,00 \\
\hline Incorreta (mochila pendendo em um só ombro) & $217(44,3)$ & $147(67,7)$ & 0,538 & $0,96(0,85$ a 1,09$)$ \\
\hline \multicolumn{5}{|l|}{ Postura em sala de aula } \\
\hline Correta (pés e costas apoiados) & $50(10,1)$ & $33(66,0)$ & & 1,0 \\
\hline Incorreta (outras) & $445(89,9)$ & $311(69,9)$ & 0,571 & $1,06(0,86$ a 1,30$)$ \\
\hline \multicolumn{5}{|l|}{ Postura ao assistir à televisão ${ }^{c}$} \\
\hline Correta (pés e costas apoiados) & $36(7,4)$ & $29(80,6)$ & & 1,00 \\
\hline Incorreta (outras) & $453(92,6)$ & $309(68,2)$ & 0,123 & $0,85(0,71$ a 1,01$)$ \\
\hline \multicolumn{5}{|l|}{ Horas assistindo à televisão por semana } \\
\hline 0 a 10 & $334(67,5)$ & $237(71,0)$ & & 1,00 \\
\hline mais de 10 & $161(32,5)$ & $107(66,5)$ & 0,309 & $0,94(0,82$ a 1,07$)$ \\
\hline \multicolumn{5}{|l|}{ Postura ao usar computador ${ }^{c}$} \\
\hline Correta (pés e costas apoiados) & $111(27,5)$ & $75(67,6)$ & & 1,00 \\
\hline Incorreta (outras) & $292(72,5)$ & $202(69,2)$ & 0,755 & $1,02(0,88$ a 1,19$)$ \\
\hline \multicolumn{5}{|l|}{ Tempo de uso do computador por semana (horas) } \\
\hline 0 a 6 & $410(82,8)$ & $284(69,3)$ & & 1,00 \\
\hline Mais de 6 & $85(17,2)$ & $60(70,6)$ & 0,810 & $1,02(0,88$ a 1,19$)$ \\
\hline
\end{tabular}

${ }^{a}$ Análise bivariada. Teste do qui-quadrado.

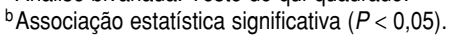

${ }^{c}$ Somente para as alunas às quais a variável se aplica.

riada ou multivariada, apenas o IMC e o número de horas assistindo à televisão apresentaram associação com as alterações posturais laterais. As adolescentes com IMC normal apresentaram maior prevalência de alterações posturais laterais se comparadas às adolescentes com sobrepeso ou obesidade. A morfologia e a velocidade de crescimento são apontadas como fatores de influência no aparecimento de alterações laterais, de modo que as adolescentes ectomorfas e aquelas que apresentam uma maior velocidade no estirão de crescimento são mais suscetíveis a tais alterações $(21,22)$. As alunas que assistiam à televisão por mais de 10 horas semanais apresentaram maior prevalência de alterações posturais laterais em relação às que não assistiam à televisão ou que assistiam à televisão por menos de 10 horas sema- nais, provavelmente devido ao fato de o tempo de exposição a essa atividade favorecer uma postura incorreta.

Em relação às demais variáveis comportamentais, não houve associação estatística com as alterações laterais. Houve uma tendência de as alunas que carregavam bolsa ou mochila de forma incorreta apresentarem alterações, porém a associação não foi significativa. Outros pesquisadores avaliaram a 
postura de escolares do ensino médio através de fotos e observaram que existe alteração na postura vista de perfil no momento do uso de mochilas, independentemente do seu peso. Porém, esses pesquisadores não estabeleceram uma relação entre a postura dos alunos com e sem a mochila (23).

Outras variáveis, como a postura ao utilizar o computador, ao sentar em sala de aula e ao assistir à televisão, não apresentaram associação estatística. Porém, observamos que a maioria das alunas adotava uma postura incorreta nessas situações. Outros autores estudaram as posturas incorretas de crianças e adolescentes no dia-a-dia, sem contudo terem realizado um estudo de associação entre essas posturas e alterações na coluna vertebral (24).

As alterações posturais ânteroposteriores apresentaram associação com as variáveis socioeconômicas, tanto na análise bivariada quanto na multivariada. As alunas de escolas públicas tiveram maior prevalência dessas alterações quando comparadas com alunas de escolas privadas. As alunas cujos responsáveis (tanto femininos quanto masculinos) estudaram apenas até o nível fundamental apresentam maior prevalência de alterações ântero- posteriores, comparadas com as alunas cujos responsáveis estudaram até o nível superior ou cursaram pós-graduação. Essa associação entre as alterações ântero-posteriores e os fatores socioeconômicos sugere que a escolaridade dos responsáveis (principalmente da responsável feminina) é de grande importância para a postura das adolescentes. Considerando-se que as alterações laterais costumam ser mais sutis, na sua aparência, do que as ântero-posteriores, é possível supor que os responsáveis tenham ficado mais atentos à postura ânteroposterior do que à postura lateral, embora não existam na literatura dados para subsidiar essa especulação. Estudos futuros devem avaliar o conheci- mento dos responsáveis sobre os cuidados com a postura corporal.

As alterações ântero-posteriores apresentaram associação com o IMC, sendo que as alunas com sobrepeso ou obesidade tiveram maior prevalência de alterações ântero-posteriores do que as alunas com IMC normal. Isso é atribuído ao fato de que o centro de gravidade do corpo é alterado em função do excesso de massa corporal, exigindo um ajuste da postura para a manutenção do equilíbrio (25).

O principal resultado deste estudo foi a alta prevalência de alterações posturais. Frente a esse resultado, é essencial que os profissionais ligados à saúde (principalmente professores de educação física, por estarem diretamente relacionados às questões corporais de seus alunos e, muitas vezes, por serem os únicos a quem os adolescentes têm acesso) façam uma avaliação completa da postura dos adolescentes. A metodologia avaliativa utilizada nesta pesquisa pode ser incluída no plano de curso dos professores de educação física ou nos exames médicos de rotina da escola (caso houver). Entendendo-se que a identificação precoce das alterações da postura corporal pode ser uma ação preventiva na área da saúde coletiva, justifica-se a inclusão de rotinas de avaliação postural na escola, uma vez que isso pode fornecer um diagnóstico inicial de alterações da coluna vertebral, que, se não identificadas precocemente, podem resultar em patologias da coluna vertebral, sendo hoje um problema de saúde pública e uma das principais causas de afastamento do trabalho $(1,26)$.

As variáveis comportamentais indicaram alta prevalência de postura sentada incorreta. Considerando que essas posturas avaliadas são mantidas por longos períodos de tempo, entende-se ser necessário um trabalho educativo no âmbito escolar para conscientizar os estudantes sobre a importância da manutenção de uma postura correta na posição sentada. Nesse sentido, outros pesquisadores realizaram um estudo com o objetivo de aplicar e avaliar um programa de educação sobre a postura correta de sentar e identificaram que o professor treinado é um importante elemento para transferir e reforçar informações sobre as situações de risco do ambiente escolar, pois o contato diário dele com o aluno favorece o ensinoaprendizagem, principalmente se o educador utilizar exercícios posturais que possibilitem aos escolares relacionar a teoria com a prática (27). Assim, pesquisas que avaliem a saúde de crianças e adolescentes são importantes tanto para o diagnóstico precoce de possíveis patologias quanto para a criação de programas de educação em saúde, principalmente nas escolas.

Em suma, entende-se que a metodologia utilizada neste estudo, bem como o rigor observado nas medições e avaliações da postura, permitiram verificar a prevalência de alterações posturais nas adolescentes escolares de uma cidade brasileira e os fatores associados a essas alterações. Os resultados mostraram uma alta prevalência de alterações nas posturas das alunas, além de revelar que essas estão associadas com variáveis socioeconômicas (tipo de escola e escolaridade dos responsáveis), antropométricas (IMC) e comportamentais (número de horas assistindo à televisão por semana). Essa alta prevalência é preocupante, tanto do ponto de vista da saúde coletiva quanto do ponto de vista ortopédico. Sendo assim, considera-se importante que os profissionais da área de saúde estejam aptos a realizar avaliações da postura, e que essas avaliações comecem a fazer parte do cronograma escolar. Com a inserção desse procedimento no âmbito escolar, alterações e patologias da coluna vertebral poderão ser identificadas precocemente. 


\section{REFERÊNCIAS}

1. Webb R, Brammah T, Lunt M, Urwin M, Allison T, Symmons D. Prevalence and predictors of intense, chronic, and disabling neck and back pain in the UK general population. Spine. 2003;28(11):1195-1202.

2. Hakala P, Rimpelã A, Salminen JJ, Virtanen SM, Rimpelã M. Back, neck, and shoulder pain in Finnish adolescents: National cross sectional surveys. BMJ. 2002;323(743):1-4.

3. Gross J, Fetto J, Rosen E. Exame musculoesquelético. Porto Alegre: Artes Médicas Sul; 2000.

4. Bunnell WP. Selective screening for scoliosis. Clin Orthop Relat Res. 2005;May(434):40-5.

5. Nissinen MJ, Heliövaara MM, Seitsamo JT, Könönen MH, Hurmerinta KA, Poussa MS. Development of trunk asymmetry in a cohort of children ages 11 to 22 years. Spine. 2000; 25(5):570-4.

6. Shehab DK, Al-Jarallah KF. Nonspecific lowback pain in Kuwaiti children and adolescents: associated factors. J Adolesc Health. 2005;36(1):32-5.

7. Detsch C, Candotti CT. A incidência de desvios posturais em meninas de 6 a 17 anos da cidade de Novo Hamburgo. Movimento. 2001;7(15):43-56.

8. Whide T. Spine: posture, mobility and pain. A longitudinal study from childhood to adolescence. Eur Spine J. 2001;10(2):118-23.

9. Brasil, Ministério da Saúde. População residente, Rio Grande do Sul. Disponível em: http://tabnet.datasus.gov.br/cgi/deftohtm. exe?ibge/cnv/popRS.def. Acessado em $1^{\circ}$ de outubro de 2004.

10. Knoplich J. Enfermidades da coluna vertebral. 2a ed. São Paulo: Panamed Editorial; 1986.

11. Grimmer K, Williams M. Gender-age environmental associates of adolescent low back pain. Appl Ergon. 2000;31(4):343-360.
12. Cole TJ, Bellizzi MC, Flegal KM, Dietz WH Establishing a standard definition for child overweight and obesity worldwide: international survey. BMJ. 2000;320(7244):1-6.

13. Kendall FP, McCreary EK, Provance PG Músculos, provas e funções. 4a ed. São Paulo: Manole; 1995

14. Santos A. Diagnóstico clínico postural: um guia prático. 3a ed. São Paulo: Summus; 2001.

15. Carneiro JAO, Sousa LM, Munaro LR. Predominância de desvios posturais em estudantes de Educação Física da Universidade Estadual do Sudoeste da Bahia. Rev Saude Com. 2005; 1(2):118-23.

16. Silva AS, Sousa MSC, Gomes ERM, Silva JMFL, Canuto PS, Neto RAA, Pontes LM. Prevalência de alterações posturais para prescrição do programa de exercícios em academias de ginástica - PB. Rev Saude Com. 2005;1(2):124-33.

17. Martelli RC, Tarebert J. Estudo descritivo das alterações posturais da coluna vertebral em escolares de 10 a 16 anos de idade. TangaráSC, 2004. Rev Bras Epidemiol. 2006;9(1): 87-93.

18. Pereira LM, Barros, PCC, Oliveira MND, Barbosa AR. Escoliose: triagem em escolares de 10 a 15 anos. Rev Saude Com. 2005;1(2): 134-43.

19. Correa AL, Pereira JS, Silva MAG. Avaliação dos desvios posturais em escolares: estudo preliminar. Fisioterapia Brasil. 2005; 6(3): $175-8$.

20. Iwamoto J, Abe H, Tsukimura Y, Wakano K Relationship between radiographic abnormalities of lumbar spine and incidence of low back pain in high school rugby players: a prospective study. Scand J Med Sci Sports. 2005;15(3):163-8.
21. LeBlanc R, Labelle H, Rivard C-H, Poitras B. Relation between adolescent idiopathic scoliosis and morphologic somatotypes. Spine. 1997;22(21):2532-6.

22. Ylikoski M. Height of girls with adolescent idiopathic scoliosis. Eur Spine J. 2003;12: 288-91.

23. Grimmer K, Dansie B, Milanese S, Pirunsan $\mathrm{U}$, Trott P. Adolescent standing postural response to backpacks load: a randomised controlled experimental study. BMC Musculoskelet Disord. 2002;3(10):1-10.

24. Cardon G, Clercq DD, Bourdeaudhuij ID, Breithecker D. Sitting habits in elementary schoolchildren: a traditional versus a moving school. Patient Educ Couns. 2004;54(2): $133-42$.

25. Fanuele JC, Abdu WA, Hanscom B, Weinstein JN. Association between obesity and functional status in patients with spine disease. Spine. 2002;27(3):306-12.

26. Alexandre NMC, Nordin M, Hiebert R, Campello M. Predictors of compliance with shortterm treatment among patients with back pain. Rev Panam Salud Publica. 2002;12(2): 86-95.

27. Zapater AR, Silveira DM, Vitta Ad, Padovani CR, Silva JCPD. Postura sentada: a eficácia de um programa de educação para escolares. Ciencia Saude Coletiva. 2004;9(1):191-9.

Manuscrito recebido em 21 de março de 2006. Aceito em versão revisada em 8 de fevereiro de 2007.
ABSTRACT

Prevalence of postural changes in high school students in a city in southern Brazil
Objective. To estimate the prevalence of lateral and anteroposterior postural changes in female adolescents and to investigate whether these changes are associated with certain socioeconomic, demographic, anthropometric, or behavioral variables.

Methods. This epidemiologic survey included a representative sample of 495 high school students from regular day school programs in the city of São Leopoldo, Rio Grande do Sul, Brazil, which is in the South region of Brazil. The students, who were 14 to 18 years old, were assessed in October and November of 2004. Postural changes were defined as skews in the spinal curvature, identified through noninvasive postural assessment.

Results. The prevalence of lateral changes was $66 \%$ (95\% confidence interval (CI): $61.5 \%$ to $70.0 \%$ ) vs. $70 \%$ for anteroposterior changes ( $95 \%$ CI: $65.2 \%$ to $73.5 \%$ ). Lateral changes were more prevalent in students with a normal body mass index (prevalence ratio $(\mathrm{PR})=1.32 ; 95 \% \mathrm{CI}: 1.09$ to 1.59 ) and in those who watched television for more than 10 hours weekly ( $\mathrm{PR}=1.16 ; 95 \% \mathrm{CI}$ : 1.02 to 1.32$)$. The prevalence of anteroposterior changes was higher in students whose parents/guardians had no schooling or only had elementary schooling (female guardians: PR $=1.30,95 \%$ CI of 1.09 to 1.55 ; male guardians: $\mathrm{PR}=1.20,95 \% \mathrm{CI}$ of 1.02 to 1.40 ) and in students who were overweight or obese (PR $=1.33 ; 95 \% \mathrm{CI}$ of 1.19 to 1.48 ).

Conclusions. The high prevalence of postural changes observed is reason for concern since these changes can translate into spinal problems in the medium to long term. Health professionals, including physical education teachers, should be trained to perform postural assessments, which should be routinely done in schools.

Key words Spine, posture, adolescent, school health services, Brazil. 